\title{
CONGRUENCE PROPERTIES \\ AND DENSITY PROBLEMS FOR THE FOURIER COEFFICIENTS OF MODULAR FORMS
}

\author{
T. HJELLE and T. KLøVE
}

\section{Introduction.}

Let

$$
\begin{aligned}
& x=e^{2 \pi i \tau}, \quad \operatorname{Im} \tau>0, \\
& \varphi(x)=\prod_{n=1}^{\infty}\left(1-x^{n}\right), \\
& \sum_{n=0}^{\infty} p_{k}(n) x^{n}=\varphi(x)^{k},
\end{aligned}
$$

where $k$ is an integer. Then $p_{-1}(n)=p(n)$ is the number of unrestricted partitions of $n$. Further, let $c(n)$ be the Fourier coefficient of Klein's modular invariant $j(\tau)$ given by

$$
j(\tau)=x^{-1} \varphi(x)^{-24}\left(1+240 \sum_{n=1}^{\infty} \sigma_{3}(n) x^{n}\right)^{3},
$$

where $\sigma_{3}(n)=\Sigma_{d \mid n} d^{3}$. Atkin and O'Brien [2] have proposed the question:

$$
\begin{aligned}
& \text { Given } a, m, \text { is } p(n) \equiv a(\bmod m) \text { soluble for } \\
& \text { values of } n \text { with positive density? }
\end{aligned}
$$

They also note that the best hope of establishing (A) is that one may exhibit explicit congruences of the form

$$
p(b n+c) \equiv a(\bmod m) .
$$

The same questions, of course, arise for $c(n)$, and indeed for the Fourier coefficients of other modular forms and functions.

In this paper we make some contribution to the solution of these problems for $p_{k}(n)$ with $k>0, p(n)$ and $c(n)$, when $a=0$.

\section{The theorems.}

Dedekind's modular form $\eta(\tau)$ is given by

$$
\eta(\tau)=e^{\pi i \tau / 12} \varphi(x) .
$$

Received June 3, 1968. 
Now, put

$$
\eta(\tau)^{k}=\sum_{n=k /(k, 24)}^{\infty} T_{k}(n) e^{(k, 24) \pi i n \tau / 12},
$$

where $k$ is an integer, and $(a, b)$ is the greatest common divisor of the integers $a, b$. The $T_{k}(n)$ 's have the following congruence property:

Theorem 1. Let k be a positive integer, and let $Q$ be a square-free number. Then, to each prime $p$ such that $p \nmid Q, p^{2} \equiv 1(\bmod 24 /(k, 24))$, there exists an even integer $M$ such that

$$
T_{k}\left(p^{m M-1} n\right) \equiv T_{k}(n / p)(\bmod Q)
$$

for all $n$ and all $m \geqq 0$.

The $T_{k}(n)$ 's are closely connected to the $p_{k}(n)$ 's, viz.

$$
\begin{aligned}
& T_{k}(n)=p_{k}(((k, 24) n-k) / 24), \\
& p_{k}(m)=T_{k}((24 m+k) /(k, 24))
\end{aligned}
$$

(see lemma 4 of Kløve [3]). As an immediate consequence of Theorem 1 we therefore have

Corollary 1.

$$
p_{k}\left(p^{m M-1} n+k\left(p^{m M}-1\right) / 24\right) \equiv 0 \quad(\bmod Q)
$$

for all $n$ prime to $p$ and all $m \geqq 1$.

Now, if $f(n)$ is any arithmetical function with integral values, put

Then corollary 1 implies

$$
d(f \mid m)=\liminf _{x \rightarrow \infty} x^{-1} \sum_{\substack{n \leq x \\ f(n) \equiv 0(\bmod m)}} 1 .
$$

COROLLARY 2.

$$
d\left(p_{k} \mid Q\right)>0
$$

for all $k \geqq 1$ and all square-free $Q$.

For $T_{-1}(n)$ we use the special notation

$$
T_{-1}(n)=P(n) .
$$

We shall prove the following congruence property of $P(n)$ (for the definition of the class of $p$-regular primes, see section 4 ):

Theorem 2. Let $Q$ be a product of different p-regular primes. Then to each prime $p$ such that $p \nmid Q, p \geqq 5$, there exists an even integer $L$ such that 


$$
P\left(p^{m L-1} Q n\right) \equiv P(Q n / p) \quad(\bmod Q)
$$

for all $n$ and all $m \geqq 0$.

By (1), $P(n)$ is connected to $p(n)$ through

$$
P(n)=p((n+1) / 24), \quad p(m)=P(24 m-1) .
$$

Thus Theorem 2 implies

Corollary 1. If $24 S \equiv 1(\bmod Q), 0<S<Q$, then

$$
p\left(p^{m L-1}(Q n+p S)-\left(p^{m L}-1\right) / 24\right) \equiv 0 \quad(\bmod Q)
$$

for all $n$ prime to $p$ and all $m \geqq 1$.

This, in turn, gives

CoRollaRY 2. If $Q$ is a product of different p-regular primes, then

$$
d(p \mid Q)>0 .
$$

In particular the primes

$$
5,7,11,13,17,19,29,31,37,41,43,53,59
$$

are $p$-regular. Now the well known Ramanujan congruences for $p(n)$ $(\bmod 5 \cdot 7 \cdot 11)$ imply $(2)$ for $Q=5 \cdot 7 \cdot 11$. Further, the results of Atkin and O'Brien [2] imply (2) for $Q=13$, and the results announced in Atkin [1] imply (2) for $Q=17 \cdot 19 \cdot 23 \cdot 29 \cdot 31$ (note that Atkin [1] has a result for the modulus 23 , which we cannot get by our methods). However, the result for $Q=37 \cdot 41 \cdot 43 \cdot 53 \cdot 59$ seems to be new.

Similarly, we shall prove the following congruence property for $c(n)$ (for the definition of the class of $c$-regular primes, see section 4):

Theorem 3. Let $Q$ be a product of different c-regular primes. Then, to each prime $p$ such that $p \nmid Q$, there exists an integer $N$ such that

$$
c\left(p^{m N-1} Q n\right) \equiv c(Q n / p)(\bmod Q)
$$

for all $n$ and all $m \geqq 0$.

An immediate consequence of Theorem 3 is

Corollary 1.

$$
c\left(p^{m N-1} Q n\right) \equiv 0 \quad(\bmod Q)
$$

for all $n$ prime to $p$ and all $m \geqq 1$.

Therefore we have 
COROLlaRY 2. If $Q$ is a product of different c-regular primes, then

$$
d(c \mid Q)>0 .
$$

In particular the primes

$$
2,3,5,7,11,13,17,19,23,29,31
$$

are $c$-regular. Now the results of Lehmer [6] imply (3) for $Q=2 \cdot 3 \cdot 5$, and the results of Lehner [7] imply (3) for $Q=7 \cdot 11$. Further, the results of Newman [8] imply (3) for $Q=13$, and lately Kolberg has proved a result which implies (3) for $Q=17 \cdot 19 \cdot 23$. However, the result for $Q=29 \cdot 31$ seems to be new.

\section{Proof of Theorem 1.}

Let $k$ be an even positive integer. Then, if $p$ is a prime such that $p^{2} \equiv 1(\bmod 24 /(k, 24))$, there exist integers $\Delta_{\alpha}$ such that $\left(\Delta_{1}, \Delta_{2}, \ldots, \Delta_{a+1}\right)$ $=1$, where $a=[(k-1) / 24]$, and

$$
\left\{\begin{array}{c}
\sum_{\alpha=1}^{a+1} \Delta_{\alpha}\left\{T_{k}\left(p^{2 \alpha} n\right)+p^{(k-2) \alpha} T_{k}\left(n / p^{2 \alpha}\right)+\right. \\
+\sum_{s=1}^{2 \alpha-1} p^{\frac{1}{2}(2 \alpha-s) k-2 \alpha}(-1)^{\frac{1}{4} k\left(p^{s-1}\right)} . \\
\left.\cdot\left\{p^{s} \delta\left(n / p^{2 \alpha-s}\right)-p^{s-1} \delta\left(n / p^{2 \alpha-s-1}\right)\right\} T_{k}\left(p^{2 s-2 \alpha} n\right)\right\} \\
=\Delta_{0} T_{l k}(n)
\end{array}\right.
$$

(Theorem 6 of Kløve [3]). Here

$$
\delta(x)= \begin{cases}1 & \text { if } x \text { is an integer }, \\ 0 & \text { otherwise }\end{cases}
$$

and $[x]$ is the largest integer in $x$. A quite similar result exists, when $k$ is an odd positive integer (Theorem 7 of Kløve [3]).

Let now $k$ be a positive integer and $q$ a given prime. Then there exists an integer $b=b(p)$ such that $\Delta_{b} \equiv 0(\bmod q)$, while $\Delta_{\alpha} \equiv 0(\bmod q)$ for $\alpha>b$. Solving (4) (or the similar equation, if $k$ is odd), we get

$$
T_{k}\left(p^{2 b} n\right) \equiv a_{1}(n) T_{k}\left(p^{2 b-2} n\right)+\ldots+a_{2 b}(n) T_{k}\left(p^{-2 b} n\right) \quad(\bmod q),
$$

where in particular $a_{2 b}(n)=-p^{(k-2) b}$. Replacing $n$ by $n p^{2 b-1}$ we obtain

$$
T_{k}\left(p^{4 b-1} n\right) \equiv a_{1} T_{k}\left(p^{4 b-3} n\right)+\ldots+a_{2 b} T_{k}\left(p^{-1} n\right) \quad(\bmod q),
$$

where now all $a_{i}=a_{i}\left(n p^{2 b-1}\right)$ are independent of $n$. This shows that for all $n$ the function $f(r)=T_{k}\left(p^{2 r-1} n\right)$ is a solution of the linear recurrence relation 


$$
f(r) \equiv a_{1} f(r-1)+\ldots+a_{2 b} f(r-2 b) \quad(\bmod q) \quad \text { for } r \geqq 2 b .
$$

Using now a well known result on linear recurrence, we conclude that if $p \neq q$ (so that $\left(a_{2 b}, q\right)=1$ ) there exists an even integer $\mu$ (independent of $n$ ) such that

$$
T_{k}\left(p^{m \mu-1} n\right) \equiv T_{k}(n / p) \quad(\bmod q)
$$

for all $n$ and all $m \geqq 0$.

Let now $q_{1}, q_{2}, \ldots, q_{r}$ be different primes, $Q=q_{1} q_{2} \ldots q_{r}$ and $p$ a prime such that $p \nmid Q, p^{2} \equiv 1(\bmod 24 /(k, 24))$. To each $q_{i}$ we associate an even integer $\mu_{i}$ given by (5). Then, with $M=\left\{\mu_{1}, \mu_{2}, \ldots, \mu_{r}\right\}$ (the least common multiple of $\left.\mu_{1}, \mu_{2}, \ldots, \mu_{r}\right)$, Theorem 1 follows.

\section{Proofs of Theorems 2 and 3.}

The following two lemmas are due to Kolberg [4], [5]:

Lemma 1. Let $q$ be a prime $\geqq 5$, and put $t=(q-1) /(q-1,12)$, $v=[(q+11) / 24]$. Then there exist constants $a_{k}$, not all $\equiv 0(\bmod q)$, such that

$$
a_{0} P(q n) \equiv \sum_{k=1}^{v} a_{k} T_{24 k t-1}(q n) \quad(\bmod q),
$$

where the sum is empty when $v=0$.

LemMa 2. Let $q$ be a prime, and put $t=(q-1) /(q-1,12), r=[q / 12]$. Then there exist constants $\alpha_{k}$, not all $\equiv 0(\bmod q)$, such that

$$
\alpha_{0} c(q n) \equiv \sum_{k=1}^{r} \alpha_{k} T_{24 k t}(q n) \quad(\bmod q),
$$

where the sum is empty when $r=0$.

If the set of integers $a_{k}$ in lemma 1 can be chosen such that $a_{0} \neq 0$ $(\bmod q)$, we define $q$ as $p$-regular. Obviously, if $q$ is $p$-regular, we get a congruence of the form

$$
P(q n) \equiv \sum_{k=1}^{v} b_{k} T_{24 k t-1}(q n) \quad(\bmod q) .
$$

Similarly, if the set of integers $\alpha_{k}$ in lemma 2 can be chosen such that $\alpha_{0} \equiv 0(\bmod q)$, we define $q$ as $c$-regular; and if $q$ is $c$-regular, we get a congruence of the form

$$
c(q n) \equiv \sum_{k=1}^{r} \beta_{k} T_{24 k t}(q n) \quad(\bmod q) .
$$

Before completing the proofs of Theorems 2 and 3 we shall give several instances of (6) and (7) (written out in the $p_{k}$-notation). We have 


$$
\begin{aligned}
p(5 n+4) & \equiv 0 \quad(\bmod 5) \\
p(7 n+5) & \equiv 0 \quad(\bmod 7) \\
p(11 n+6) & \equiv 0 \quad(\bmod 11)
\end{aligned}
$$

These are the cases of (6) with $v=0$ and are recognized as the well known Ramanujan congruences. Further

$$
\begin{aligned}
p(13 n+6) & \equiv 6 p_{23}(13 n+5) \quad(\bmod 13) \\
p(17 n+5) & \equiv p_{95}(17 n+1) \quad(\bmod 17) \\
p(19 n+4) & \equiv p_{71}(19 n+1) \quad(\bmod 19) \\
p(29 n+23) & \equiv 7 p_{167}(29 n+16) \quad(\bmod 29) \\
p(31 n+22) & \equiv 22 p_{119}(31 n+17) \quad(\bmod 31) .
\end{aligned}
$$

These are the cases of (6) with $v=1$ and are given by Kolberg [4]. Further

$$
\begin{aligned}
& p(37 n+17) \equiv p_{71}(37 n+14)+19 p_{143}(37 n+11) \quad(\bmod 37), \\
& p(41 n+12) \equiv 35 p_{239}(41 n+2)+3 p_{479}(41 n-8) \quad(\bmod 41), \\
& p(43 n+9) \equiv 23 p_{167}(43 n+2)+5 p_{335}(43 n-5) \quad(\bmod 43), \\
& p(53 n+42) \equiv 8 p_{311}(53 n+29)+14 p_{623}(53 n+16) \quad(\bmod 53), \\
& p(59 n+32) \equiv 27 p_{695}(59 n+3)+58 p_{1391}(59 n-26) \quad(\bmod 59) .
\end{aligned}
$$

These are the cases of (6) with $v=2$ and seem to be new.

Similarly, we have

$$
\begin{aligned}
& c(2 n) \equiv 0 \quad(\bmod 2), \\
& c(3 n) \equiv 0 \quad(\bmod 3) \text {, } \\
& c(5 n) \equiv 0 \quad(\bmod 5) \text {, } \\
& c(7 n) \equiv 0 \quad(\bmod 7) \\
& c(11 n) \equiv 0 \quad(\bmod 11) \text {, } \\
& c(13 n) \equiv 8 p_{24}(13 n-1) \quad(\bmod 13), \\
& c(17 n) \equiv 7 p_{96}(17 n-4)(\bmod 17) \text {, } \\
& c(19 n) \equiv 4 p_{72}(19 n-3)(\bmod 19) \text {, } \\
& c(23 n) \equiv 13 p_{264}(23 n-11)(\bmod 23) \text {, } \\
& c(29 n) \equiv 4 p_{168}(29 n-7)+23 p_{336}(29 n-14)(\bmod 29) \text {, } \\
& c(31 n) \equiv p_{120}(31 n-5)+25 p_{240}(31 n-10)(\bmod 31) \text {. }
\end{aligned}
$$

The above results for $q=2$ and 3 are implied by the congruence $(n+1)$ $c(n) \equiv 0(\bmod 24)$ given by Lehmer [6]. The other cases of (7) with $r=0$ are implied by the congruences of Lehner [7], and the cases of (7) with $r=1$ are given by Kolberg [5] (the congruence $c(13 n) \equiv-\tau(n)(\bmod 13)$ of Newman [8], where $\tau(n)$ is Ramanujan's function, implies the above result for $q=13$, as noted by Kolberg [5]). The cases of (7) with $r=2$ seem to be new. 
The necessary computation for establishing (6) and (7) in the cases with $v=2$ and $r=2$ was performed by the second author on the IBM $360 / 50$ computer at the University of Bergen.

We now turn to the proof of Theorem 2 . Let $q \geqq 13$ be a $p$-regular prime, and let $p$ be a prime $\neq q$ such that $p^{2} \equiv 1(\bmod 24 /(24 k t-1,24))$ $(k=1, \ldots, v)$, that is, $p \geqq 5$. To each of the functions $T_{24 k t-1}(n)(k=1, \ldots, v)$ we associate an even integer $\mu_{k}$ given by (5). Put $\Lambda=\left\{\mu_{1}, \mu_{2}, \ldots \mu_{v}\right\}$; then we have

that is,

$$
\begin{aligned}
P\left(p^{m \Lambda-1} q n\right) & \equiv \sum_{k=1}^{v} b_{k} T_{24 k t-1}\left(p^{m \Lambda-1} q n\right) \\
& \equiv \sum_{k=1}^{v} b_{k} T_{24 k t-1}(q n / p) \quad(\bmod q)
\end{aligned}
$$

$$
P\left(p^{m \Lambda-1} q n\right) \equiv P(q n / p) \quad(\bmod q) .
$$

If $q \leqq 11$ is a $p$-regular prime, then $P(q n) \equiv 0(\bmod q)$, so that $(8)$ is obvious for any integral $\Lambda$.

Let now $q_{1}, q_{2}, \ldots, q_{r}$ be different $p$-regular primes, $Q=q_{1} q_{2} \ldots q_{r}$ and $p$ a prime such that $p \nmid Q, p \geqq 5$. Put $L=\left\{\Lambda_{1}, \Lambda_{2}, \ldots \Lambda_{r}\right\}$, where $\Lambda_{i}$ is an even integer associated to $q_{i}$ through (8), and Theorem 2 follows.

Starting from lemma 2, Theorem 3 is proved in a similar way.

\section{REFERENCES}

1. A. O. L. Atkin, Multiplicative concruence properties and density problems for $p(n)$, Proc. London Math. Soc. (3) 18 (1968), 563-76.

2. A. O. L. Atkin and J. N. O'Brien, Some properties of $p(n)$ and $c(n)$ modulo powers of 13, Trans. Amer. Math. Soc. 126 (1967), 442-459.

3. T. Kløve, Recurrence formulae for the coefficients of modular forms and congruences for the partition function and for the coefficients of $j(\tau),(j(\tau)-1728)^{\frac{1}{2}}$ and $(j(\tau))^{\frac{1}{3}}$, Math. Scand. 24 (1968), 133-169.

4. O. Kolberg, Some remarks on a class of partition congruences, Årbok Univ. Bergen, Mat.-Naturv. Ser. 1961, No. 18.

5. O. Kolberg, Congruences for the coefficients of the modular invariant $j(\tau)$, Math. Scand. 10 (1962), 173-181.

6. D. H. Lehmer, Properties of the coefficients of the modular invariant $J(\tau)$, Amer. J. Math. 64 (1942), 488-502.

7. J. Lehner, Divisibility properties of the Fourier coefficients of the modular invariant $j(\tau)$, Amer. J. Math. 71 (1949), 136-148.

8. M. Newman, Congruences for the coefficients of modular forms and for the coefficients of $j(\tau)$, Proc. Amer. Math. Soc. 9 (1958), 609-612. 